Journal of Computer Science 7 (2): 234-241, 2011

ISSN 1549-3636

(C) 2011 Science Publications

\title{
Drawing Meshed One-Line Diagrams of Electric Power Systems using a Modified Controlled Spring Embedder Algorithm Enhanced with Geospatial Data
}

\author{
Alexandre de Assis Mota and Lia Toledo Moreira Mota \\ Exact, Environmental and Technological Sciences Center, \\ Pontifical Catholic University of Campinas, Brazil
}

\begin{abstract}
Problem statement: One-line diagrams are widely used in electrical power systems to provide a graphical view of state of the system and to assess its performance. Several methodologies and algorithms have been proposed to automatically draw the power system's one-line diagram and provide satisfactory results for radial or small-size meshed networks. Nevertheless, actual power systems models are generally associated to strongly meshed networks and in this case, the usage of the mentioned methodologies can yield unsatisfactory solutions, with a great number of line crossings. Approach: This study proposes a method that generates bus/branch one-line diagrams of meshed power systems, based on a modified version of the Controlled Spring Embedder algorithm enhanced by the usage of physical laws and geospatial data. Results: The methodology was tested using a portion of the Brazilian interconnected power system that corresponds to a meshed bus/branch model with 58 nodes and 64 branches. The graphical portrayal was obtained from the branches list of the network, firstly without the presence of geographical information and then considering it. Conclusion: The proposed algorithm was able to evolve a graphical portrayal of the bus/branch electric model of a power system, yielding satisfactory results. In special, geospatial data have proved to be fundamental information, allowing the elimination of branch crossings and providing consistent representations for a specific electrical network in $95 \%$ of the simulated cases.
\end{abstract}

Key words: One-line diagrams, power system, electric power system, graph layout, controlled spring embedder algorithm, geospatial data, scientific visualization, bus/branch model, graph drawing, power system monitoring, Spring Embedder (SE), Rooted Tree (RT)

\section{INTRODUCTION}

One-line diagrams are widely used in a great number of power systems analysis functions (e.g., power flow, state estimation, contingency analysis and voltage sensitivity analysis), yielding an overview of the current state of the system and permitting the assessment of its performance under specific operational conditions. Particularly in simulations and studies, the bus/branch oneline diagram model can be considered as the most important form to represent the electric network (Monticelli, 1999; Arganis and Domínguez, 2008; Kumkratug, 2010a; Taher and Besharat, 2008).

Up to date, many efforts have been made in order to develop techniques to visualize the electrical network and its associated variables, as shown in the references (Sun and Overbye, 2004; Mota et al., 2002; Rao and Deekshit, 2004; Mota et al., 2007). Nevertheless, all these methods take into account that the one-line diagram graphical representation is already available or that it can be manually built using CAD (Computer Aided Design) tools, as the electric network portrayals illustrated in (Sun and Overbye, 2004; Li and Chiang, 1999; Mota et al., 2002; Prabha et al., 2009). However, if the large dimension of actual power systems is considered, the application of this kind of manual technique can become impracticable. Besides, there are some network studies (as the contingency analysis) that imply in many simulations with significant topological changes in the system. In this case, all these modifications are reflected in the one-line diagrams and must be represented through different networks. Using the mentioned CAD tools, every alteration in the network topology must be manually set, making very difficult its utilization in this type of analysis. Consequently, the development of methodologies to automatic generate and visualize these diagrams is of fundamental importance.

Corresponding Author: Alexandre de Assis Mota, Centro de Ciências Exatas, Ambientais e de Tecnologias Pontifícia Universidade Católica de Campinas, Rodovia D. Pedro I, km 136, Parque das Universidades, Campinas -SP-Brasil, CEP 13086-900 Tel: +55-19-37567370 
Several methodologies and algorithms have been proposed to automatically draw power systems' oneline diagrams. References (Sun and Overbye, 2004; Mota et al., 2002; Battista et al., 1999; Rao and Deekshit, 2004; Mota et al., 2007; Ong et al., 2000; Mota et al., 2007) show successful applications of these methods. These results illustrate graphical representations of radial networks, which commonly correspond to distribution systems, or of small-size meshed systems. However, large transmission power systems are generally associated to strongly meshed networks and, depending on the studies that need to be made in the system, the associated bus/branch model can significantly vary in topology, number of branches and number of nodes. In this case, the usage of the mentioned methodologies can yield unsatisfactory solutions, generating a great number of line crossings. In this context, this study proposes a method that generates bus/branch one-line diagrams of strongly meshed power systems, based on physical laws and enhanced by the usage of geospatial data.

\section{MATERIALS AND METHODS}

Generation of one-line diagrams with graph layout techniques: The topology of a bus/branch one-line diagram can be associated to the connections of an undirected graph. In this case, the buses and branches of the diagram can be respectively related to the nodes and arcs of a graph. So, this correspondence makes possible the usage of graph layout techniques (graph drawing methods) to generate the one-line diagram of an electric system. This problem can be reduced to the generation of a graph with minimal crossings, based on the connectivity information among the buses (network topology data). Regarding the bus/branch one-line diagram model, this information can be extracted from the system nodal admittance matrix (Monticelli, 1999; Mota et al., 2002; Kumkratug, 2010a; 2010b), where diagonal elements define the buses and non-diagonal elements define the branches. Though, considering interconnected and large actual power systems (with thousands of nodes and arcs), automatic graph drawing is not a simple task.

Fundamental efforts were recently made to develop or apply methods that permit the one-line diagram automatic generation ( $\mathrm{Li}$ and Chiang, 1999; Rao and Deekshit, 2004; Ong et al., 2000; Mota et al., 2005; Prabha et al., 2009). Specially, reference (Ong et al., 2000) presents an important performance comparison among three graph algorithms (Spring Embedder, Rooted Tree and Controlled Spring Embedder) that automatically yield one-line diagrams layout. The
Spring Embedder (SE) algorithm is characterized by the drawing of one-line diagrams in a total automatic way, with no user intervention. This aspect can be considered as an advantage in some specific studies, but for the main users (the control center operators), it can represent a limitation of the algorithm, since the graphical portrayal, automatically generated, can significantly differ from the layout desired by the operators. The Rooted Tree (RT) algorithm allows some user intervention, but due to its peculiar characteristics, certain system operators may reject the final graph layout. This can occur since the algorithm always generate a root element (root bus) on the top of the portrayal and all other buses are located in the draw in a top-down or left-right fashion. Finally, the Controlled Spring Embedder (CSE) algorithm is a modified version of the Spring Embedder algorithm that permits user interaction. In this case, the user is responsible for a lot of decisions, fixing and dragging buses and this can lead to a very slow generation of the diagram.

Despite the disadvantages of these algorithms, all of them are able to automatically draw one-line diagrams of radial networks or of weakly meshed systems. However, transmission power systems are in fact continental interconnected networks that correspond to strongly meshed systems. In this context, this study proposes a methodology to automatically draw the bus/branch one-line diagram of this type of network (strongly meshed), based on one of the previously mentioned algorithms: the Controlled Spring Embedder. The selection of this algorithm was founded on the comparison among the advantages and disadvantages related to SE, RT and CSE algorithms. This comparison shows that in spite of CSE disadvantages, it is able to generate one-line diagrams with good readability (for weakly meshed networks) and yield great user satisfaction due to its high level of intervention. Nevertheless, in order to make its application to strongly meshed power systems viable, some crucial changes in the original CSE algorithm were needed. These alterations generate a modified version of the previously mentioned CSE algorithm: the Enhanced Controlled Spring Embedder algorithm (ECSE), described in the following.

Enhanced controlled spring embedder algorithm: The original CSE algorithm models the graph nodes as graph components connected by springs. Initially, these nodes are placed in a pre-determined position and, then, the interactions among the springs lead the graph nodes' positions to a state of "minimum energy". This state is the final graphical representation of the one-line diagram that corresponds to the definitive placement of 
all graph elements (buses and branches). This state can be achieved through the definition of attractive and repulsive forces between pairs of components connected by a branch (or spring). Equations (1) and (2) define these forces:

$F_{a}=\frac{d^{2}}{k}$

$\mathrm{F}_{\mathrm{r}}=\frac{-\mathrm{k}^{2}}{\mathrm{~d}}$

Where:

$\mathrm{F}_{\mathrm{a}}=$ The attractive force between neighboring components

$F_{r}=$ The repulsive force between every pair of components and

$\mathrm{D}=$ The actual distance between two graph nodes

The user variable $\mathrm{k}$ is the desired ideal distance between components, because when $\mathrm{d}$ is equal to $\mathrm{k}$, the sum of the attractive and repulsive forces cancels each other.

These forces must be determined for each graph element. However, the user can define fixed components that will not be subjected to forces computation. Hence, for these components, $F_{a}=F_{r}=0$.

When applied to strongly meshed systems, the CSE algorithm presents some problems related to a fundamental requirement of graph drawing algorithms: the readability of the generated graphical representation. A diagram is readable if its meaning can be well inferred from the way it is drawn (Ong et al., 2000). So, changes in the original CSE algorithm were implemented to enhance this requirement. The next subsections present all the implemented modifications.

Spring length: In the conventional CSE algorithm, the distance between two graph nodes is defined by $\mathrm{d}$. Nevertheless, the distance between the nodes in the graph representation corresponds to the spring length and consequently to the branch length in the one-line diagram. So, there is a correspondence between these quantities and this fact can be used to enhance the readability of the generated one-line diagram. The ECSE algorithm, proposed in this study, suggests the definition of springs' lengths according to the natural length of the corresponding branches. Hence, if the user defines these lengths, the generated graphical portrayal can become more similar to the desired one, enhancing the user satisfaction.

So, the force $F_{H}$ associated to a pair of adjacent buses (A and B) can be determined using Eq. (3).

$\mathrm{F}_{\mathrm{H}}=\mathrm{K}_{\mathrm{H}} \cdot\left(\mathrm{L}-\sqrt{\left(\mathrm{x}_{\mathrm{a}}-\mathrm{x}_{\mathrm{b}}\right)^{2}+\left(\mathrm{y}_{\mathrm{a}}-\mathrm{y}_{\mathrm{b}}\right)^{2}}\right)$
In (3), $\mathrm{K}_{\mathrm{H}}$ and $\mathrm{L}$ (natural branch length) are constants defined by the user, $\{x a, y a\}$ and $\{x b, y b\}$ are, respectively, the coordinates of the adjacent buses A and $\mathrm{B}$ and the force $\mathrm{F}_{\mathrm{H}}$ is similar to the one that arises in a spring when stretched or compressed, given by the Hooke's law. Consequently, the spring force in the ECSE algorithm can present attractive or repulsive nature.

Charged nodes: The charged nodes correspond to another important modification in the CSE algorithm proposed in this study, because they also provide enhancements in the readability of the generated oneline diagram. In the ECSE algorithm, a force between any pair of buses (not only adjacent buses) can be defined as in Eq. 4.

$\mathrm{F}_{\mathrm{C}}=\frac{\mathrm{K}_{\mathrm{C}}}{\lambda+\left(\mathrm{x}_{\mathrm{a}}-\mathrm{x}_{\mathrm{b}}\right)^{2}+\left(\mathrm{y}_{\mathrm{a}}-\mathrm{y}_{\mathrm{b}}\right)^{2}}$

In (4), $\{x a, y a\}$ and $\{x b, y b\}$ are, respectively, the coordinates of buses $\mathrm{A}$ and $\mathrm{B}$ and the force $\mathrm{F}_{\mathrm{C}}$ is similar to the electrostatic force between two punctiform bodies with charges of same polarity (given by the Coulomb's law). $\lambda$ is a non-null constant defined by the user, particularly important when considering the usage of geospatial data, as will be described in the following. $\mathrm{K}_{\mathrm{C}}$ is a user-defined constant that emulates hypothetical electrical charges $\mathrm{Q}_{A}$ and $\mathrm{Q}_{B}$ associated to the nodes.

Iterative node displacement and user intervention: The objective of the ECSE algorithm is to automatically generate the one-line diagram of a strongly meshed system. Since the one-line diagram is a graphic representation with only two dimensions, this goal can be achieved by finding the coordinates $\left\{\mathrm{x}_{\mathrm{i}}, \mathrm{y}_{\mathrm{i}}\right\}$ associated to the location of each bus i. Then, the branches can be depicted using these coordinates as references. If random initial values for the coordinates are adopted, an iterative sequence of adjustments must be made to find an optimal position for each bus, based on the application of Eq. 3 and 4.

These adjustments are made in terms of horizontal and vertical displacements $\Delta \mathrm{x}_{\mathrm{i}}$ and $\Delta \mathrm{y}_{\mathrm{i}}$ for the coordinates $x_{i}$ and $y_{i}$, of each node $i$ in the network. For a force $F$ between two buses $\mathrm{A}$ and $\mathrm{B}$, these displacements can be determined, at each iteration, by Eq. 5 and 6 :

$$
\begin{aligned}
& \Delta \mathrm{x}_{\mathrm{A}}=\frac{\left(\mathrm{x}_{\mathrm{a}}-\mathrm{x}_{\mathrm{b}}\right)}{\sqrt{\left(\mathrm{x}_{\mathrm{a}}-\mathrm{x}_{\mathrm{b}}\right)^{2}+\left(\mathrm{y}_{\mathrm{a}}-\mathrm{y}_{\mathrm{b}}\right)^{2}}} \cdot \mathrm{F} ; \Delta \mathrm{x}_{\mathrm{B}}=-\Delta \mathrm{x}_{\mathrm{A}} \\
& \Delta \mathrm{y}_{\mathrm{A}}=\frac{\left(\mathrm{y}_{\mathrm{a}}-\mathrm{y}_{\mathrm{b}}\right)}{\sqrt{\left(\mathrm{x}_{\mathrm{a}}-\mathrm{x}_{\mathrm{b}}\right)^{2}+\left(\mathrm{y}_{\mathrm{a}}-\mathrm{y}_{\mathrm{b}}\right)^{2}}} \cdot \mathrm{F} ; \Delta \mathrm{y}_{\mathrm{B}}=-\Delta \mathrm{y}_{\mathrm{A}}
\end{aligned}
$$


After iteration starts, $\mathrm{F}$ assumes the values of forces $\mathrm{F}_{\mathrm{H}}$ and $\mathrm{F}_{\mathrm{C}}$, in Eq. 5 and 6. That means the displacements must be determined twice for each pair of nodes: firstly using $\mathrm{F}_{\mathrm{H}}$ and then using $\mathrm{F}_{\mathrm{C}}$, in the previous equations. All displacements are calculated for all nodes. The nodes graphical coordinates are then adjusted, using the net value for the displacement in each node and the graphical portrayal of the network is updated. The iteration is then finished and, if necessary, another one can begin.

This iterative process proceeds until all the incremental adjustments are smaller than a predefined tolerance or a maximum number of iterations is reached. During this iterative procedure, the ECSE algorithm allows the user intervention to fix the graph components. This is also a modification that contributes to enhance the one-line diagram readability, since the conventional CSE algorithm does not include the possibility of this intervention along its processing.

Usage of geospatial data: As pointed before, the CSE algorithm has a disadvantage, when compared to other graph layout techniques: the time needed to generate the diagram is significantly increased due the need of user interaction. In this case, the user makes most of the decisions about the graphical location and mobility of the network components through mouse clicking and dragging operations.

In order to minimize the time required to obtain the final graphical representation, the amount of user interaction must be reduced. In this study, this goal was achieved by linking geographical location (geospatial information) to the electrical data, generating corresponding graphical coordinates that can be used as an initial point for the ECSE algorithm. This association is direct, since each element (bus or branch) has a nonambiguous correspondence to a physical system apparatus. The system apparatus, in their turn, possess an unique geographic position. These locations can be derived, for instance, from the worldwide radionavigation system Global Positioning System (GPS).

Just as the systems apparatus, each network component also possesses a unique geographic position. However, one can encounter some difficulties in obtaining the geospatial data related to all network components, especially in large electric power systems. In this situation, the location of the system facilities (that correspond to groups of apparatus and equipment) can be used to determine the coordinates of singular components. For example, the geospatial data of a substation can be used to localize all of its internal buses, if there is no information about the individual location of these buses.

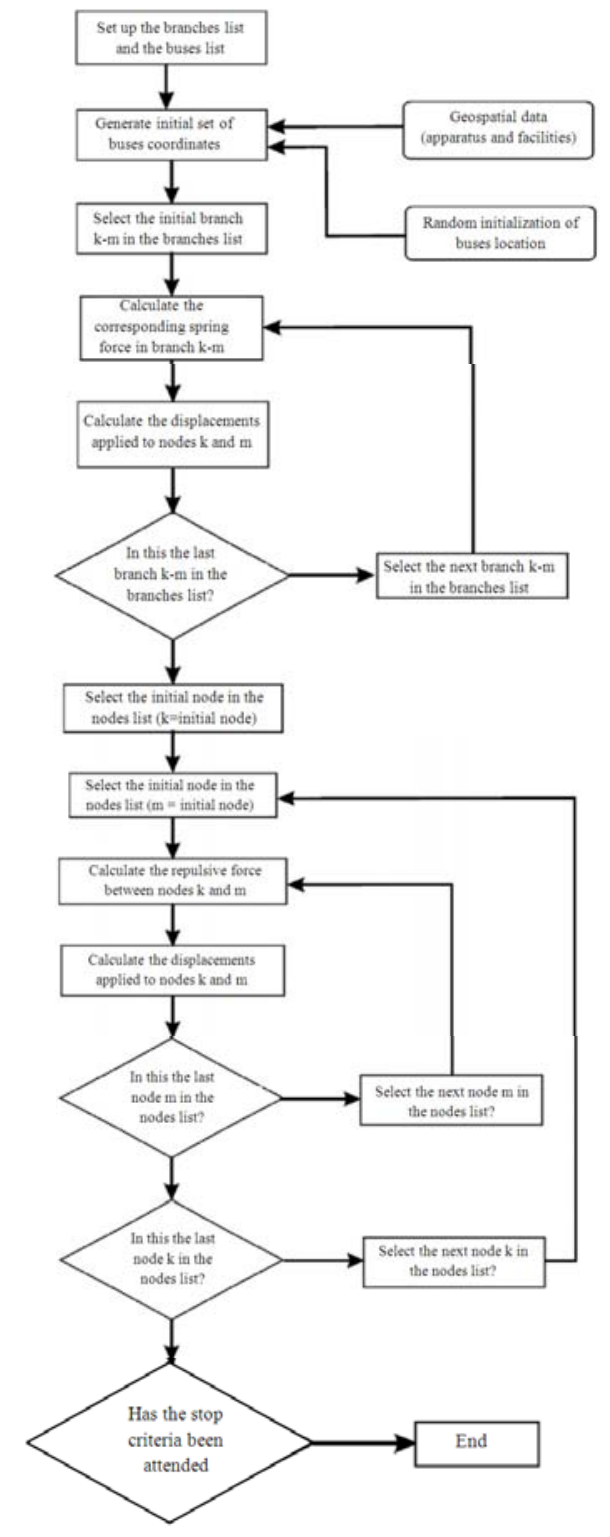

Fig. 1: Nodes placement algorithm

By adopting this approach, the initial network representation will present coincidences in the location of some buses. In this case, the conventional CSE algorithm will present a severe problem related to the forces among the networks elements, for equations that depend on the distance between buses cannot be determined. By other hand, the proposed ECSE algorithm can provide a solution for these forces when the distance between a pair of elements is null, by the usage of the constant $\lambda$ previously defined in Eq. 4. That makes the usage of geospatial data feasible with the ECSE algorithm, even when there are overlapped buses. 


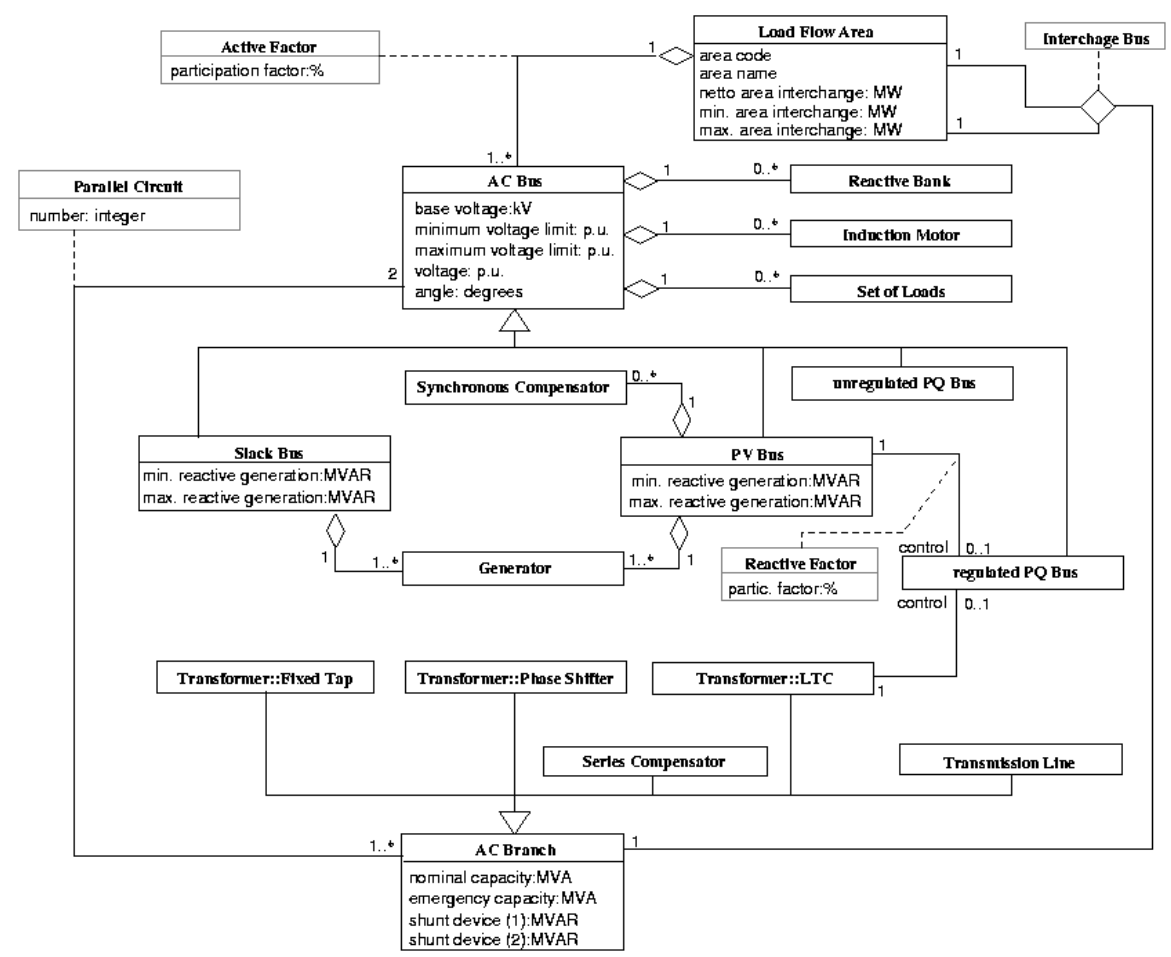

Fig. 2: Conceptual data model of the transmission system components considering their bus/branch representation

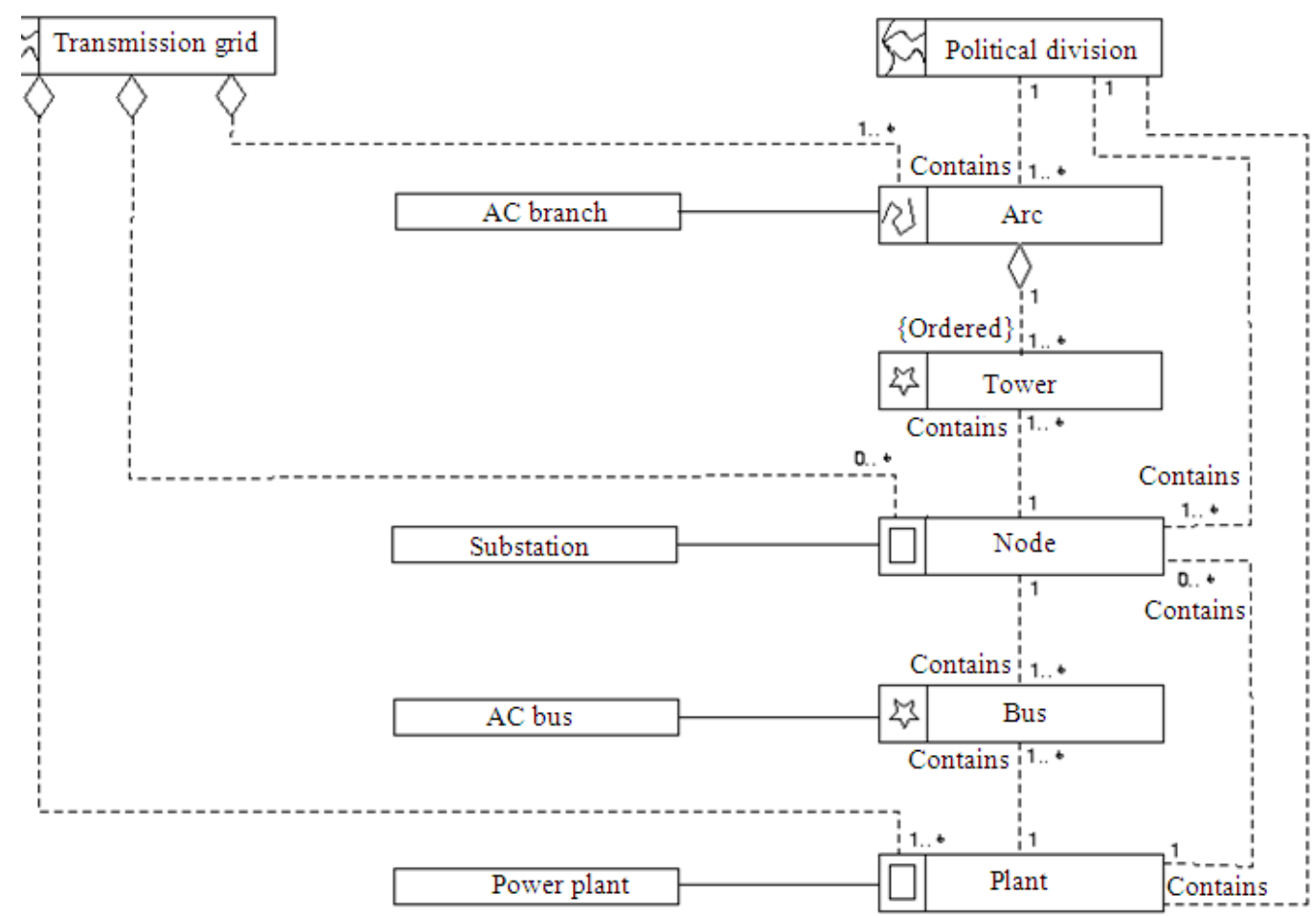

Fig. 3: Conceptual data model of the relations among the transmission system components and geospatial information 
The ECSE complete algorithm is represented in Fig. 1 and it is related to the buses placement stage of the one-line diagram generating process. After this stage, the diagram can be prepared for line routing.

In this context, several efficient routing algorithms can be used, such as the Lee and Maze algorithms and the HighTower algorithm (Ong et al., 2000; Hoel et al., 1976), so, in this study, the results focus in the placement of the network nodes.

Outline of the approach: The following steps summarize the methodology proposed in this study:

Step A: Obtain the topology (connections relations) of the power systems elements (buses and branches). The conceptual data model of the power system components adopted in this study considering the bus/branch representation, obtained from reference (Mota et al., 2005), is shown in Fig. 2.

Step B: Add to this topology information, geospatial data related to the network elements (substations, towers), as shown by the conceptual data model (Mota et al., 2005) illustrated in Fig. 3.

Step C: Application of ECSE algorithm to automatically generate the one-line diagram.

\section{RESULTS}

The proposed algorithm was executed on a portion of the Brazilian interconnected power system. The branches list associated to this network is shown in Fig. 4.

As can be inferred from the branches list, 58 nodes and 64 branches compose this system's bus/branch network. Without geospatial information, the corresponding graph presents several branch crossings and superposition of nodes, as shown in Fig. 5. Also, there is a multiplicity of one-line representations, when different executions of the visualization algorithm are considered, e.g., the user obtain a different graphic representation of the network every time the algorithm is executed.

When the algorithm is executed considering geospatial information for the buses, the results are better, even when the data is available only for a few buses, as presented in Table 1 .

In this case, the initial representation, in the presence of geospatial information, is shown in Fig. 6.

As expected, nodes that represent network buses that are in the same geographical region were drawn as superposed bars. However, due the physical laws that govern the algorithm, after a few iterations the graphic representation of the network becomes as shown in Fig. 7. 100 simulations were made with this subsystem. In the presence of geospatial data, the graphic representation converged to one similar to the network shown in Fig. 7 in $95 \%$ of all results obtained from the algorithm executions.
Table 1: Geospatial information provided for the algorithm

\begin{tabular}{ll}
\hline Geographical region & Buses \\
\hline Northwest & $5651,5652,5653$ \\
North-central & $5458,5428,5411$ \\
Northeast & $5621,5401,5413$ \\
Southwest & 5452,5453 \\
South-central & 5421 \\
Not available & All the remaining buses \\
\hline
\end{tabular}

\section{Brazilian sub-system branches list}

5401-5402; 5401-5403; 5401-5411; 5403-5406; 5408-5428; 5411-5408; 54115410; 5411-5418; 5411-5419; 5411-5421; 5411-5621; 5411-5631; 5413-5417; $5416-5417$; 5418-5412; 5418-5413; 5419-5413; 5419-5414; 5421-5422; 5421$5431 ; 5421-5441 ; 5421-5452 ; 5421-5471 ; 5423-5421 ; 5423-5424 ; 5424-5426$; $5428-5451 ; 5431-5433 ; 5431-5441 ; 5441-5443 ; 5441-5461 ; 5443-5445$; 5443$5446 ; 5452-5450 ; 5452-5453 ; 5452-5471 ; 5452-5651 ; 5453-5454 ; 5453-5456$; $5453-5473 ; 5454-5455 ; 5456-5457 ; 5458-5451 ; 5458-5452 ; 5458-5641 ; 5458-$ $5651 ; 5471-5473 ; 5621-5623 ; 5623-5624 ; 5631-5421 ; 5631-5633 ; 5641-5643$ (1) $5651-5481 ; 5651-5652 ; 5651-5653 ; 5651-5667 ; 5651-5668$; 5652-5654; 5652$5656 ; 5667-5660 ; 5667-5661 ; 5668-5662 ; 5668-5663 ; 7029-5653$;

Fig. 4: Brazilian sub-system branches list

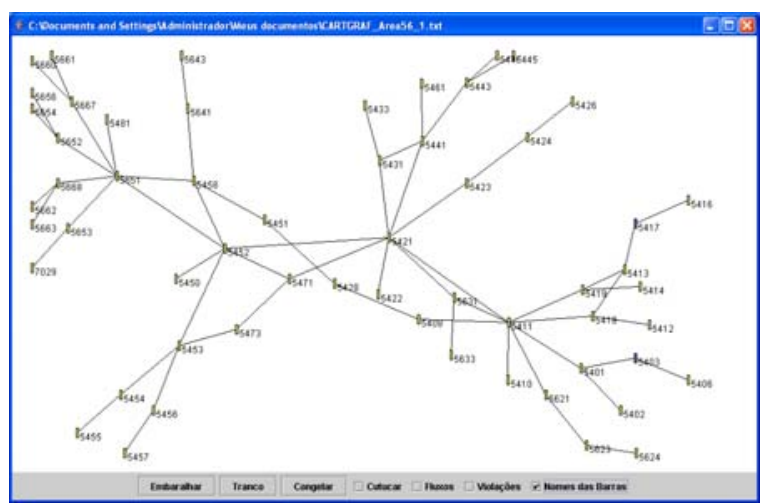

Fig. 5: Network diagram generated without geospatial information

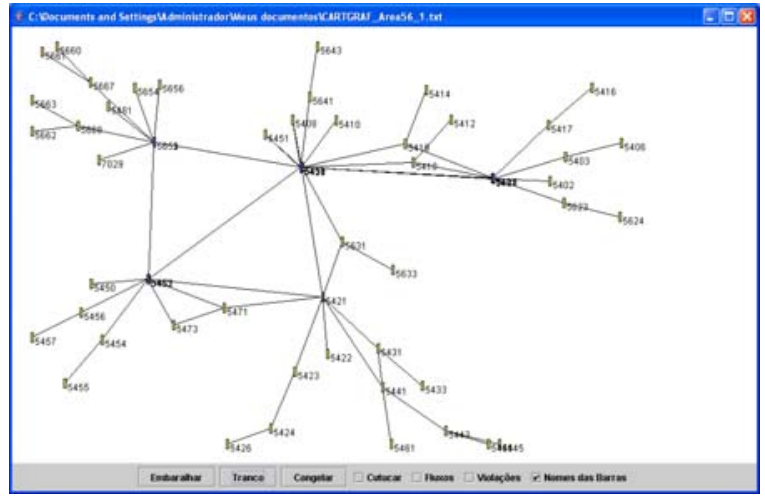

Fig. 6: Brazilian sub-system one-line diagram generated with geospatial information-initial representation 


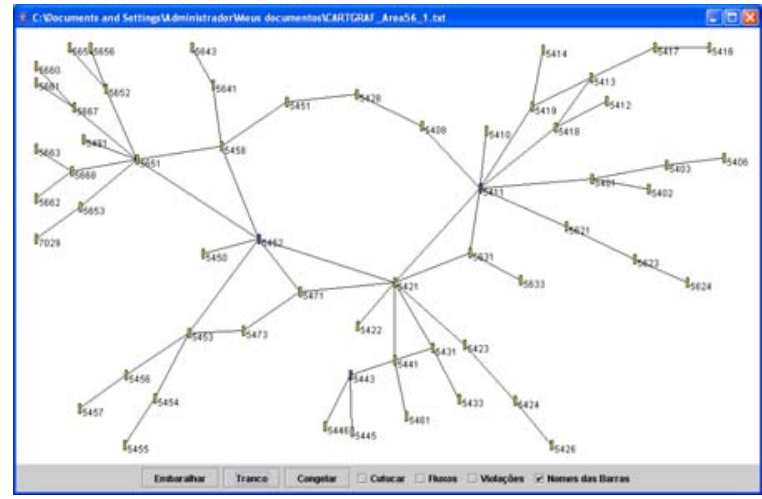

Fig. 7:Brazilian sub-system one-line diagram generated with geospatial information-final representation

\section{DISCUSSION}

It can be noted that in initial representations using geospatial data, several branch crossings occur, because nodes graphical coordinates are randomly initialized. Consequently, there is also a multiplicity of one-line representations provided by the algorithm, i.e., it yields a different diagram every time it is executed.

The performance of the algorithm using geospatial data, however, is satisfactory even when the geographical location was made available only for a few buses. So, the method is efficient when there is lack of geospatial information.

In the results presented, the algorithm has eliminated all the branch crossings in the final diagram with geospatial information available for only 12 busesthat corresponds to $20 \%$ from the total of buses. Also, it was able to evolve to one consistent representation for different random initializations.

\section{CONCLUSION}

Nowadays, electrical power systems play a critical role in people's life, as evidenced by the recent major blackouts in the world. All the supervision, maintenance and studies related to electric power generation and transmission are strongly based on network models represented by one-line diagrams. The complexity and size of actual electrical power systems makes these diagrams automatic generation and corresponding updates, very complicated tasks. Many efforts have been made to apply graph visualization methods to the confection of these diagrams; however, the usage of these techniques can become inefficient due to the presence in the final portrayal of many branch crossings. Also, there is the problem of the generation of multiple graphical representations for a same electric network.
In this context, geospatial data have proved to be fundamental information, allowing the complete elimination of crossings in many cases, as demonstrated by the results of this study. Its usage has also the benefit of consistent diagram generation for a specific power system model, improving the similarity among graphical representations generated in different executions of the ECSE algorithm.

\section{REFERENCES}

Arganis, M.L. and R. Domínguez, 2008. Hydropower system management considering the minimum outflow. Am. J. Environ. Sci., 4: 178-184. DOI: 10.3844/ajessp.2008.178.184

Battista, G., P. Eades, R. Tamassia and I.G. Tollis, 1999. Graph Drawing: Algorithms for the Visualization of Graphs. 1st Edn., Prentice Hall, USA., ISBN: 0133016153, pp: 397.

Hoel, J.H., H.B. Gooi and C.K. Chan, 1976. Some Variations of Lee's algorithm. IEEE Trans. Comput, 25: 19-24. DOI: 10.1109/TC.1976.5009200

Kumkratug, P., 2010a. Application of interline power flow controller to increase transient stability of power system. J. Comput. Sci., 6: 1490-1493. DOI: 10.3844/jcssp.2010.1484.1487

Kumkratug, P., 2010b. Fast decoupled power flow for power system with high voltage direct current transmission line system. Am. J. Applied Sci., 7: 1115-1117. DOI: 10.3844/ajassp.2010.1115.1117

Li, H. and H.D. Chiang, 1999. One-line diagram builder for power system analysis. Proceeding of the IEEE Power Engineering Society Summer Meeting, July 18-22, Edmonton, Alta. , Canada, pp: 652-657. DOI: 10.1109/PESS.1999.787394

Monticelli, A., 1999. State Estimation in Electric Power Systems-A Generalized Approach. 1st Edn., Springer, USA., ISBN-10: 0792385195, pp: 416.

Mota, A.A., L.T.M. Mota and A. Monticelli, 2002. Animated User Interfaces for State Visualization in Electric Power System Operations. IEEE PES Transmission and Distribution Latin America, Sao Paulo, Brazil. http://www.geoinfo.info/geoinfo2005/papers/P14.PDF

Mota, A.A., L.T.M. Mota and A. Morelato, 2007. Visualization of power system restoration plans using CPM/PERT graphs. IEEE Trans. Power Syst., 22: 1322-1329. DOI: 10.1109/TPWRS.2007.901118 
Mota, A.A., L.T.M. Mota and W.S. Ting, 2005. Using geospatial data to generate one-line diagrams of electrical power systems. http://citeseerx.ist.psu.edu/viewdoc/summary?doi= 10.1.1.144.3122

Ong, Y.S., H.B. Gooi and C.K. Chan, 2000. Algorithms for automatic generation of one-line diagrams. IEE Proceed. Generation Transmission Distribution, 147: 292-298.

Prabha, S.U., B.S. Dayasagar and C. Venkataseshaiah, 2009. Visualization of available power transfer capability in a transmission system using morphological techniques. Am. J. Applied Sci., 6: 247-250. DOI: 10.3844/ajassp.2009.247.250
Rao, P.S.N. and R. Deekshit, 2004. Distribution feeder one-line diagram generation: a visibility representation. Electric. Power Syst. Res., 70: 173178. DOI: 10.1016/j.epsr.2003.12.005

Sun, Y. and T.J. Overbye, 2004. Visualizations for power system contingency analysis data. IEEE Trans. Power Syst., 19: 1859-1866. DOI: 10.1109/TPWRS.2004.836193

Taher, S.A. and H. Besharat, 2008. Transmission congestion management by determining optimal location of FACTS devices in deregulated power systems. Am. J. Applied Sci., 5: 242-247. DOI: 10.3844/ajassp.2008.242.247 